\title{
A Survey of Intelligent Transportation Systems
}

\author{
Eng. Woroud A. Alothman* \\ $\mathrm{PhD}$ Researcher in Mechanical Engineering, College of Engineering, University of Bahrain, Manama, \\ 00973, Kingdom of Bahrain. \\ E.mail: woroudalothman@gmail.com \\ Prof. Saad Suliman \\ Professor of Manufacturing Processes and Systems, College of Engineering, University of Bahrain, \\ Kingdom of Bahrain. \\ Prof. Hashim N. Al-Madani \\ Professor of Transportation and Civil Engineering, College of Engineering, University of Bahrain, \\ Kingdom of Bahrain.
}

\begin{abstract}
The problem of traffic delay and congestion is a severe problem in the world due to the population growth and difficulties in changing the infrastructure. This issue is being studied by researchers and international traffic centers because the traffic problem affected by the delay in services and products and the negative economic that traffic problem causes. It is difficult to improve the traffic system performance by using the traditional control methods. Many studies had been conducted using the fuzzy logic system and neural network to control the road intersections. In this article, the artificial intelligence traffic control principles and approaches which applied in the traffic signal control are reviewed. A comparison between the artificial intelligent systems and some points of view about future research in this area are proposed. The review shows that the traffic performance of the fuzzy controller has better performance than traditional traffic signal controls, especially during heavy and uneven traffic volume conditions.
\end{abstract}

Keywords - Artificial Intelligence, Fuzzy Logic, Intelligent Transportation Systems, Neural Networks, Traffic Control Systems.

\section{Introduction}

Traffic congestion and delay problems appear in many cities due to the increasing demand for the vehicle and transportation systems. This caused a loss of travel time and economic cost. Artificial Intelligence (AI) techniques are used to solve these types of problems. This strategy may improve road capacity, traffic light performance and reduce vehicle delay by adjusting parameters such as cycle, splits, phase sequences and offsets per change of the traffic volume. The most famous and important artificial traffic control systems are fuzzy, and neural network traffic light control systems. The existing applications of AI in traffic control system are fuzzy logic, artificial neural networks, and reinforcement learning algorithms controls. This artificial intelligent is a new knowledge where there is a lack of understanding in some countries of how to plan, proceed, implement, operate or benefit from this type of control system. Due to this lack of understanding, a poor maintenance of equipment was faced. The limitation of technical capacity can also be an issue. The existing traffic in the world systems are not effective in the performance, cost, and maintenance effort, where the traffic and delay problems appear in the world. Whereas, the intelligent traffic control system is more effective by increasing the number of vehicles on the road, making a way for emergency cases and reducing the lost time based on realistic traffic situations. In 2015, Shah et al. [1] proposed a fuzzy control system for the simple four phases intersection to change the phase time when vehicles are passing across and to reduce waiting time. The performance measured showed a high improvement in the control system. Whereas, Jin et al. (2016) [2] developed a fuzzy intelligent traffic control system for an isolated four phases simple intersection to improve traffic movement based on its ability in generating flexible phase structures and making intelligent timing decisions. This paper will discuss and summaries the Fuzzy Logic Traffic Control Systems and Artificial 
Neural Network Traffic Control Systems. Some points will be highlighted for future researchers.

\section{Fuzzy Logic Traffic Control System}

Fuzzy logic control is a tool used for processing complex, non-linear and non-deterministic traffic control problems. It provides an intelligent green interval response based on actual traffic situation. The mathematical control models are difficult and complicated methods, where the fuzzy control systems are used to model expert's thinking based on the actual situations by using defective information efficiently. The fuzzy control systems are used to reduce the complexity of control systems and can be implemented in hardware, software, or a combination of both. The control process is shown in Fig.1.

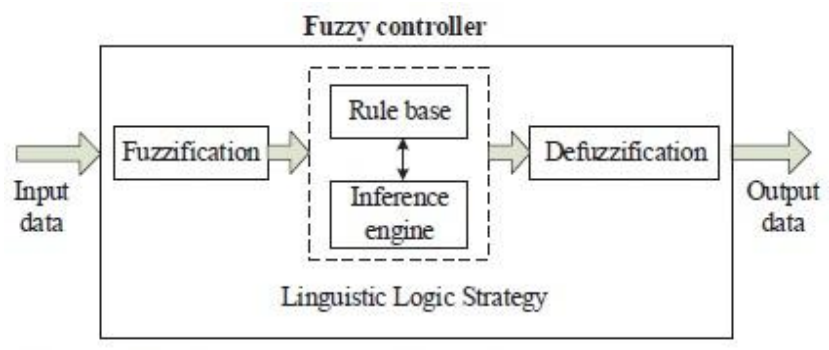

Fig.1: fuzzy control traffic system diagram [3]

During the last ten years, many studies had been conducted using fuzzy logic systems as follows:

In 2007, Fahmy [4] proposed a fuzzy logic control system for a roundabout with four approach intersection to extend or terminate the signal phase and to select the sequence of next phases based on the traffic flow that detected at the input of the fuzzy controllers and converted into fuzzy values. Performance analysis showed that the total waiting time in the developed control system is low compared with the actual system. Also, the proposed traffic system provided reliability and independence in decision making. Also, in 2007, Madhavan and Cai [5] proposed a fuzzy traffic control system to control traffic flows under ordinary and unexpected traffic situations for the isolated intersection. The developed system is receiving information from sensors that placed at incoming and outgoing lanes to make optimal decisions and to minimize the traffic delay. A simulator has been developed to evaluate the performance of the fuzzy system under different conditions. The fuzzy system showed better performance in up normal conditions and a similar performance in normal condition with existing control system. Whereas, in 2007, Vonglao [6] proposed a fuzzy logic system to calculate the best green time of each intersection phase at each cycle based on the traffic density for $\mathrm{T}$, simple and roundabout intersections. The results showed that the developed control system is more efficient than a predictable controller

In 2009, Abbas et al. [7] developed a fuzzy logic control algorithm to control traffic signals for flexible traffic on oversaturated isolated intersections with the integration of left and right turns. The developed control system was used to decide whether to extend the current green time or terminate it based on a set of fuzzy rules and real-time traffic information. It was also used to control the continuous and safe flow of emergency vehicles. Also, Zarandi and Rezapour [8] proposed a fuzzy signal control system for the T-isolated intersection with twoway streets and left-turn lanes. The control system helped in the decision to extend or terminate the current green time and to change the sequence level based on the real-time traffic information. The performance of the proposed system was evaluated by using simulation methods. The results of the developed control system showed significant improvement over pre-timed control strategy.

In 2010, Andrade et al. [9] designed a fuzzy control system for the isolated intersection located in arterial networks to control the phase green time and the average cycle time for each intersection. Furthermore, the authors studied the impact of controlling the isolated intersection on other intersections. Average delay per vehicle, the percentage of stops, and the number of stops per vehicle were used to evaluate the developed control system performance. Also, Soh, Rhung and Sakran [10] developed a fuzzy control system for isolated signalized intersection based on the waiting time and vehicle queue length at the phase green time and the vehicle queue lengths at other intersection phases. The results showed that the developed control system had a higher performance for controlling the traffic flow than the traditional methods.

In 2011, Askerzade and Mahmud [11] developed a fuzzy logic traffic control system for the simply isolated intersection to compute the optimal extension time to be added to the fixed traffic time concerning the situation of its backward and forward closer intersections. Also, in 2011, Barzegar [12] developed an intelligent fuzzy control system for an isolated intersection with two directions only to minimize the stop time based on vehicle congestion by giving time for passing vehicles in each phase and processes to change or to not change the traffic situation. Whereas, Mehan [13] proposed a fuzzy traffic control system for a simply isolated intersection to improve the traffic capacity and to reduce the delay by using the traffic density and queue length and by 
adjusting the green, yellow and red time for the actual situation. Moreover, Zaied and Alothman [14] proposed a fuzzy logic traffic system for two two-way intersections to reduce vehicle delay by adjusting changes in time intervals of a traffic signal based on traffic situation level. The results showed that the proposed system accelerated the cycle time and gave other phases the chances to gain more benefit from the green lost time.

In 2012, Hou et al. [15] developed a fuzzy traffic control system with full countdown function to solve the randomness problem and to control the green time by predicting the traffic data for a simple four phases intersection. The developed system produced a time distribution by studying and analyzing the traffic flow. The results showed that the developed control system improved the traffic flow and reduced the waiting time and saturation situation. Also, in 2012, Kuang and Xu [16] proposed a transit-priority fuzzy neural network control system. The system is proposed for a simple four phases isolated intersection to reduce the delays of passengers and special vehicles by increasing the green time of the special phase that has a red light and has a special vehicle's arrival. Once the special vehicles pass through, the next phase is selected based on the normal priority. Moreover, Letia and Cuibus [17] developed a fuzzy control system to improve the control performance by getting the optimal control decisions and by extending or terminating the phase green time for fixed order intersection phases and fixed off sets between intersections in the network. Whereas, Ma et al. (2012) [18] proposed a flexible multi-phase fuzzy control system in coordination with a genetic algorithm for a simple intersection to optimize the fuzzy membership functions based on the traffic condition at different times to be used in various traffic states. Adding to that, Mwangi et al., 2012 [19] proposed a fuzzy logic control system for the isolated roundabout intersection to control traffic light timing and phase sequence. The system is proposed to ensure smooth traffic flow, to decrease traffic delays and to increase the intersection capacity by deciding whether to extend or to terminate the current phase green time and by selecting the appropriate phase sequence based on the traffic data that collected from upstream and downstream detectors. In 2012, Zade and Dandekar [20] developed a fuzzy inference traffic control system to control the traffic green time based on traffic density and traffic flow rate by observing the ongoing traffic situation around the intersection. Moreover, in 2012, Zang et al. [21] developed an intelligent fuzzy control algorithm system for a multiphase simple signalized intersection to help in making full use of green time by optimizing green time extension of the current phase and choosing the sequence of the next phase based on the vehicle queue. The developed control system performance was measured under different traffic conditions.

In 2013, Pour et al. [22] developed a fuzzy traffic light controller to optimize the control of fluctuating traffic volumes in oversaturated or unusual load conditions for an individual T-intersection and to consider the uncertainty in the entrance and exits rates of an intersection. The control system was developed to minimize the total traffic queue and pedestrian delays. Moreover, the authors introduced a new defuzzification method to solve traffic problem and for more accuracy of calculations. Moreover, Stotsko et.al [23] developed and implemented a fuzzy algorithm-based traffic control system for the existing traffic conditions to reduce the average and maximal vehicle queue lengths for the simply isolated intersection.

In 2014, Alam, and Pandey [24] developed a fuzzy control system for simply isolated intersections based on the number of vehicles entered the phases by extending the phases green time to reduce the traffic congestion and to avoid the green time loss on an empty road. The results indicated that the developed control system has a potential to improve operations at intersections.

Also, in 2014, Ge [25] proposed a fuzzy control system for a single four phases simple intersection to specify the next phase to switch based on the urgency degree that was calculated through the red phases. The average vehicle delays were used to measure the control system performance. Whereas, Mahesh et al. [26] designed a neuro-fuzzy control system for a four phases intersection to reduce intersection delays by providing variation in the green cycle intervals based on the heavy traffic roads.

\section{Artificial Neural Network Control System}

Artificial neural network (ANN) had been developed in 1940 's. It is an important type of network system that is developed based on the human brain neural structure through receiving information, storing them and combining them to solve problems. ANN system consists of inputs, which are multiplied by weights, and then manipulated by a mathematical function. The ANN system determines the activation of the neuron beside computing the output of the artificial neuron.

In 2008, Wannige, and Sonnadara [27] developed an adaptive neuro-fuzzy system to reduce the vehicle waiting time and the vehicle delay by specifying the 
traffic green time using several inputs in the calculation such as a gap between two vehicles, delay at intersections, vehicle density, flow rate and queue length.

In 2009, Singh et al. [28] developed an artificial neural network control system to provide the optimum green time extension for all four phases, and to optimize the total number of vehicles in a road, and to identify the importance of the road in the intersection based on the real signal time.

In 2010, Anfilets and Shuts [29], developed a neural network control system to provide the traffic cycle time by calculating the changes in the traffic flows. Moreover, Arel et al. [30] produced a feedforward neural network control system to minimize the average delay, congestion, and a likelihood of intersection crossblocking by evaluating the value function approximation for scheduling traffic signals at intersection networks. Also, Çetiner et al. [31] proposed an artificial neural network system to inform the drivers about the traffic volume during the next coming hours by predicting the traffic volume based on one-year historical data that were collected earlier.

In 2011, Dunne and Ghosh [32] developed an artificial neural network control system to predict short-term traffic flow and traffic speed, where the traffic flow and speed notes from uncongested (linear) and congested (non-linear) rules are regime-adjusted. Whereas, Hodge et al. [33] developed a dual neural network algorithm to determine and apply the traffic control interferences by predicting traffic flow for a short term. Also, Pamula [34] developed a neural network system to optimize the control strategy by considering online the intensity, queue length, and by estimating road infrastructure throughput.

In 2012, Cortez et al. [35] developed a neural network to forecast the traffic by using a real traffic data in different time scales. Also, Mohammadi et al. [36] used a neural network system to find the optimum cycle time and green time for each access point of the intersection by identifying the average vehicle speed at an access point, the average waiting time and the queue length. Whereas, Sathiyavathy [37] proposed a self-adaptive neural network to predict the traffic volume. The developed system has low computational complexity, fast convergence speed, and high goodness-of-fit.

Moreover, in 2013, Royani et al. [38] applied a fuzzy neural network with expert knowledge on a real-time traffic signal for the simple four phases isolated intersection to improve the traffic control of fluctuating traffic volumes in oversaturated and unusual load condition and to improve the vehicular throughput and minimize delays. Also, in 2013 Sehrawat et al. [39] developed a new intelligent traffic management model that based on Turing machine with the application of neural network. The developed model studies the situation of its signal's traffic along with the traffic situation of its next signals to find the time ratio for each signal to reduce traffic congestion by extending the green time and ensuring a steady flow of traffic in a wide region.

In 2014, Abdul Kareem et al. [40] designed an intelligent control system using the structure of a neural network. The controller uses virtual data and information about the traffic condition of each street in the intersection. The smart decisions are taken when the intersection management determines each street case at the intersection based on collected data. The proposed system has been applied and tested in two intersections then compared with the actual control system of these intersections.

In 2015, Teja and Das [41] developed a fuzzy artificial neural network for isolated intersection and bridges to reduce delays and increase vehicular output. The developed control system was used for optimal control of irregular traffic densities during oversaturated condition. Also, Yasar and Rashid [42] developed an intelligent dynamic traffic light controller with the support of artificial neural networks algorithm for a simple four phases intersection to calculate sets of drive orders for traffic signal lights in special traffic conditions.

In 2016, Jomaa [43] integrated and applied an artificial control system includes a neural network, fuzzy logic, genetic algorithm, and simulation model to optimize traffic flow in coordinated traffic lights systems and to minimize the traffic congestion by adjusting the traffic light time. Also, Rizwan et al. [44] developed an artificial neural network to improve the traffic movement by predicting and adjusting the timing of the signals on both intersection sides of the road at the same time based on the traffic density.

\section{Conclusions}

This paper has surveyed the application of artificial intelligence to control and manage traffic systems. It is observed that the implementation of artificial intelligence in transportation systems has a substantial impact on traffic levels. Although traffic control systems 
still have some limitations, future research has a lot of promise for smart traffic control systems. Researchers are working to find ways to overcome these limitations to make them completely efficient. From the previous review, the following highlights can be made:

\section{A. Fuzzy Logic Traffic Control Systems:}

- Traffic fuzzy systems had different patterns, which are often recurrent; improve flow rate, forecast traffic capacity, control phase selection, and control signal timing;

- Most of the researchers used Mamdani type inference and depended on historical data to compare between the proposed and existing systems;

- Many researchers work at the control of an isolated intersection with fuzzy control method (Madhavan and Cai [2007], Abbas et al. [2009], Zarandi and Rezapour [2009], Andrade et al. [2010], Soh, Rhung and Sakran [2010]);

- Few of them work in a simply isolated intersection (Askerzade and Mahmud [2011], and Mehan [2011];

- Few of them work in a roundabout intersection (Fahmy [2007], and Mwangi et al. [2012])

- Some of them work in T-intersection (Vonglao [2007], Zarandi and Rezapour (2009), and Zang et al. [2012]);

- Others work in two two-way intersections (Zarandi and Rezapour [2009], and Zaied and Alothman [2011]);

- Few researchers apply a fuzzy logic system to the coordinated control of arterial or area traffic;

- Finally, the results showed that the traffic performance of Fuzzy Logic Traffic Control Systems has better performance than traditional traffic signal controls, specifically during heavy and uneven traffic volume conditions.

\section{B. Artificial Neural Network Traffic Control Systems:}

- ANNs are attractive for applications with no or little explicit knowledge about a system;

- ANNs are used for control and analysis of complex traffic systems, emergency warning, and optimization;

- The control of the traffic light, an ANN will be trained from measurement data of the traffic density over time. There are lots of methods in technical literature how to create and train an ANN;
- It is possible to optimize existing traffic light systems through artificial neural networks;

- Finally, the results showed that ANNS offer a chance to handle the increasing traffic demand. They are cost-effective, robust and can be easily implemented and upgraded to existing traffic control systems.

\section{References}

[1] Shah K., Jha J., Zala M. and Khetra N., "Improvement of Traffic Monitoring System by Density and Flow Control for Indian Road System using IoT", International Journal for Scientific Research \& Development, vol. 3, issue 10, pp. 167 170, India, 2015.

[2] Jin J., Ma X., and Konsonen I., An Intelligent Control System for Traffic Lights with Simulation-Based Evaluation, Finland, 2016.

[3] Rojas M., Ponce P. and Molina A., Novel Fuzzy Logic Controller based on Time Delay Inputs for a Conventional Electric Wheelchair, México, 2014

[4] Maged M. Fahmy M., "An Adaptive Traffic Signaling for Roundabout with Four-Approach Intersections Based on Fuzzy Logic", Journal of Computing and Information Technology, vol. 1 pp. 33 - 45, Saudi Arabia, 2007.

[5] Madhavan B. and Cai J., "A Fuzzy Logic Controller for Isolated Signalized Intersection with Traffic Abnormality Considered", Intelligent Vehicles Symposium, vol.1, Turkey, 2007

[6] Vonglao P., The Solution of Traffic Signal Timing by Using Traffic Intensity Estimation and Fuzzy Logic, Australia, 2007

[7] Abbas S., Sheraz S. and Noor H., Fuzzy Rule-Based Traffic Signal Control System for Oversaturated Intersections, Pakistan, 2009.

[8] Hossein M., Zarandi F., and Rezapour S., "A Fuzzy Signal Controller for Isolated Intersections", Journal of Uncertain Systems, vol. 3, no. 3, pp. 174 - 182. UK, 2009.

[9] Andrade M., Jacques M. and Ladeira M., Exploratory Studies on Network Operation of Fuzzy Signal Controllers, Portugal, 2010.

[10] Soh A., Rhung L., and Sarkan H., "MATLAB Simulation of Fuzzy Traffic Controller for Multilane Isolated Intersection", International Journal of Computer Science and Engineering, vol. 2, no. 4, pp. 924 - 933, Malaysia, 2010.

[11] Askerzade and Mahmud M., "Design and Implementation of Group Traffic Control System Using Fuzzy Logic", www.arpapress.com, vol. 6, issue 2, Turkey, 2011.

[12] Barzegar B., "Fuzzy Logic Controller for Traffic Signal Controller Unit System and Modeling with Colored Petri Net", Indian Journal of Science and Technology, vol. 4, no. 11, Iran. 2011.

[13] Mehan S., "Introduction of Traffic Light Controller with Fuzzy Control System", International Journal of Electronics and Communication Technology, vol. 2, issue 3, India, 2011.

[14] Zaied A. and Alothman W., "Development of a Fuzzy Logic Traffic System for Isolated Signalized Intersections in the State of Kuwait", Expert Systems with Applications, vol. 38, pp. 9434 -9441, Bahrain, 2011.

[15] Hou R., Wang Q., Wang J., Lu Y., and Kim J., "A Fuzzy Control Method of Traffic Light with Countdown Ability", International Journal of Control and Automation, vol. 5, no. 4, China, 2012 
[16] Kuang X., and Xu L., "Real-Time Traffic Signal Intelligent Control with Transit-Priority", Journal of Software, vol. 7, no. 8, China, 2012.

[17] Letia T. and Cuibus O., Cooperative Control for Urban Vehicle Traffic, Romania, 2012

[18] Ma W., Geng D., and Yan Y., "Multi-Phase Fuzzy Control of Single Intersection in Traffic System Based on Genetic Algorithm", International Journal of Innovative Computing Information and Control, vol. 8, no. 5A, pp. 3387 - 3397, 2012

[19] Mwangi C., Kangethe S. and Nyakoe G., Design and Simulation of a Fuzzy Logic Traffic Signal Controller for A Signalized Intersection, Kenya, 2012.

[20] Zade R. and Dandekar D., "Simulation of Adaptive Traffic Signal Controller in MATLAB Simulink Based on Fuzzy Inference System", National Conference on Innovative Paradigms in Engineering \& Technology, India, 2012.

[21] Zang L., Zhu W., Hu P., and Junce Li, "Study on Intelligent Control Algorithm for Traffic Signals at Multi-Phase Intersections", Journal of Computational Information Systems, vol. 8, pp. 10477 - 10484, China, 2012.

[22] Pour N., Asadi H., and Kheradmand M., "Fuzzy MultiObjective Traffic Light Signal Optimization", Journal of Applied Mathematics, vol. 2013, pp. 1 - 7, Iran, 2013.

[23] STOTSKO Z., FORNALCHYK Y., and MOHYLA I., "Simulation of Signalized Intersection Functioning with Fuzzy Control Algorithm", Transport Problems, vol. 8, issue 1, pp. 5 - 16, Ukraine, 2013.

[24] Alam J., and Pandey M., "Development of Intelligent Traffic Light System Based on Congestion Estimation Using Fuzzy Logic", Journal of Computer Engineering, vol. 16, issue 3, pp. 36 - 44, India, 2014.

[25] Ge Y., "A Two-Stage Fuzzy Logic Control Method of Traffic Signal Based on Traffic Urgency Degree", Hindawi Publishing Corporation Modelling and Simulation in Engineering, vol. 2014, pp. 1 - 6, China, 2014.

[26] Ramteke K., Nikalaje J., and Rane D., "Implementation of Intelligent Traffic Signal Controller Based on Neuro-Fuzzy System", International Journal of Engineering and Computer Science, vol. 3, issue 3, pp. 5013-5016, India, 2014.

[27] Wannige C. and Sonnadara D., "Traffic Signal Control Based on Adaptive Neuro-Fuzzy Inference", IEEE International Conference on Information and Automation for Sustainability, vol. 4, pp. 301-306, Colombo, 2008.

[28] Singh L., Tripathi S., and Arora H., "Time Optimization for Traffic Signal Control Using Genetic Algorithm", International Journal of Recent Trends in Engineering, vol 2, no. 2, India. 2009.

[29] Anfilets S., Shuts V., "Artificial Neural Networks for Adaptive Management Traffic Light Objects at the Intersection", Proceedings of the 10th International Conference Reliability and Statistics in Transportation and Communication, Latvia, 2010.

[30] Arel T., Liu C., Urbanik T., and Kohls A., "Reinforcement learning-based multi-agent system for network traffic signal control", IET Intelligent Transport Systems, vol. 4, issue 2, pp. 128-135, USA, 2010.
[31] Gültekin B., Sari M. and Borat O., "A Neural Network-Based Traffic-Flow Prediction Model", Mathematical and Computational Applications, vol. 15, no. 2, pp. 269-278, Turkey, 2010.

[32] Dunne S. and Ghosh B., Traffic Flow Predictions Employing Neural Networks in A Novel Traffic Flow Regime Separation Technique, Ireland, 2011.

[33] Hodge V., Krishnan R., Jackson T., Austin J., and Polak J., Short-Term Traffic Prediction Using a Binary Neural Network, UK, 2011.

[34] Pamula T., "Road Traffic Parameters Prediction in Urban Traffic Management Systems Using Neural Networks", Problem Transport, vol. 6, issue 3, pp. 123 - 128, Poland, 2011.

[35] Cortez P., Rio M., Rocha M. and Sousa P., "Multi-scale Internet traffic forecasting using neural networks and time series methods", Expert Systems, vol. 29, no. 2, pp. 143-155., 2012.

[36] Fazli S., Mohammadi S., and Rahmani M., "Neural Network based Vehicle Classification for Intelligent Traffic Control", International Journal of Software Engineering and Applications, vol.3, no.3, Iran, 2012.

[37] Sathiyavathy P., and Tech M., "Road Traffic Prediction Based on Self-Adaptive Neural Network", International Conference on Computing and Control Engineering, Chennai, 2012.

[38] Royani T., Haddadnia J. and Alipoor M., "Control of Traffic Light in Isolated Intersections Using Fuzzy Neural Network and Genetic Algorithm", International Journal of Computer and Electrical Engineering, vol. 5, no. 1. Iran, 2013.

[39] Sehrawat R., Malviya H. and Kaul V., "Implementation of Neural Network with a variant of Turing Machine for Traffic Flow Control", International Journal on Computer Science and Engineering, vol. 5, no. 05, pp. 343 - 348, India, 2013.

[40] Abdul Kareem E., Abbas S., and Salman S., " Intelligent traffic light controller based on MCA associative memory", Science Journal of Circuits, Systems and Signal Processing, vol. 3, no. 6-1, 2014, pp. 6-16, Iraq, 2014.

[41] Teja B. and Das R., "Fuzzy Artificial Neural Networks and Particle Swarm Optimization Based Enhanced Traffic Signal Controlling System", International Journal of Advanced Research in Electrical, vol. 4, issue 9, pp. 7656 - 7661, India, 2015.

[42] Yasar M. and Rashid M., "Implementation of Dynamic Traffic Light Controllers Using Artificial Neural Networks to Diminish Traffic Ordeals", IEEE European Modelling Symposium, Bangladesh, 2015.

[43] Jomaa K., "An Artificial Intelligence Techniques and Simulation Model to Control a Traffic Jam System in Malaysia (Review Paper)", Asian Journal of Business and Management, vol. 4, issue 1, pp. 27 - 34, Malaysia, 2016.

[44] Rizwan J., Krishnan P., Karthikeyan R. and Kumar S., "MultiLayer Perception Type Artificial Neural Network based Traffic Control", Indian Journal of Science and Technology, vol. 9, issue 5, pp. $1-6$, India, 2016. 